\title{
Focus on Diabetic Retinopathy (DR) and MicroRNA “What Association"
}

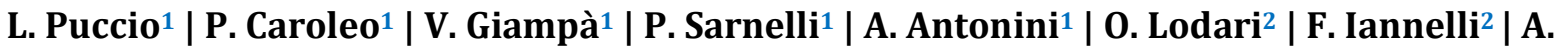 Cerchiaro² | R. Paone $^{2}$ | C. Paone ${ }^{2 *}$}

*Correspondence: Claudia Paone

Address: ${ }^{1}$ Hospital “Pugliese-Ciaccio” of Catanzaro, Italy; ${ }^{2}$ University of Studies "Magna Graecia” of Catanzaro, Italy e-mail $\bowtie$ : cla.31@libero.it

Received: 15 June 2021; Accepted: 01 July 2021

Copyright: (C) 2021 Puccio L. This is an open-access article distributed under the terms of the Creative Commons Attribution License, which permits unrestricted use, distribution, and reproduction in any medium, provided that the original work is properly cited.

\section{ABSTRACT}

Diabetes Mellitus (DM) is one of the most common chronic diseases in the world. According to the latest data from the International Diabetes Federation, it is estimated that around 700 million will develop DM by 2045!

Glycemic control is crucial in the management of DM and for the prevention of microvascular "nephropathy, retinopathy, neuropathy" and macrovascular complications. Of all the microvascular complications, the one that aroused most interest in our descriptive study was Diabetic Retinopathy (DR), since, if left untreated, it leads to irreversible anatomical and functional damage. DR is the leading cause of blindness in working-age adults across the world. The pathogenesis of DR is multifactorial and the molecular mechanisms are still not fully understood. The treatment of DR essentially involves laser therapy, which is not always decisive due to absence and/or limited availability of successful drug therapies for these blinding disorders. The clinical diagnosis of DM often occurs late and this delay means that the chronic vascular complications associated with the disease are already present at the time of diagnosis. Therefore, this risk can be reduced if an early diagnosis is made. Hence the need to identify new specific biomarkers that can act as diagnostic and prognostic indicators of the disease.

According to some studies, putative roles of small endogenous molecules of microRNAs as indicators of DR and associated complications are being considered. They carry out their silencing activity on a wide range of transcripts deriving from the expression of thousands of genes.

There is evidence for the aberrant expression of microRNAs implicated in the onset of numerous pathologies. Since most of the genetic variations associated with diabetes are found in regions of the human genome that are not coding for proteins, it is worth studying those microRNAs involved in DR, both as possible therapeutic targets and as specific predictive diagnostic biomarkers. The present study aims to highlight the importance of some microRNAs and indicate those most implicated in the onset of DR, according to the literature, for the purpose of making a previous diagnosis of diabetes, in particular of DR.

Keywords: Diabetic Retinopathy, MicroRNA, Diabetes, Retinopathies

Introduction

The term "Retinopathies" refers to a large group of pathologies affecting the retina and greater attention should be paid as they can cause loss of vision. In terms of incidence, in our society, the main diseases affecting the retina concern: age-related macular degeneration, pigmentary retinopathy, macular edema and DR (Mayur Choudhary and Goldis Malek., 2016). 
The retinopathies are characterized by an alteration of the ocular vascular component, with greater expression of different factors by retinal endothelial cells including the endothelial vascular growth factor (Jack Kanski and Brad Bowling, 2015). In fact, VEGF is responsible for a series of pro angiogenic effects characterized by vasodilation, by the increase in the permeability of blood vessels, by the release of proteolytic enzymes that invade and condition tissue remodeling with prevention of endothelial apoptosis (Yun-Zheng, 2017). Diabetes mellitus is an important cardiovascular risk factor characterized by elevated plasma glucose levels.

High glucose (HG) negatively influences endothelial cell (EC) function, which is characterized by the inability of ECs to respond to vascular endothelial growth factor (VEGF-A) stimulation (Masuda et al., 2017).

DR is a chronic, progressive disease, potentially dangerous for retinal microvascularization, associated with pathophysiological changes intensified by diabetes. DR is the most frequent and the most important ocular complication of Diabetes Mellitus (DM), characterized by more or less extensive damage to the retina and represents, according to data from the World Health Organization (WHO), one of the main causes of low vision/blindness in adults (Das and McGuire, 2003). The prevalence of DR is directly related to the duration of diabetes: after twenty years of diabeic disease, almost all patients with DMT1 and more than $70 \%$ of patients with DMT2 develop a certain degree of DR (Lee et al., 2015; Yau et al., 2012). As the global prevalence of diabetes increases, the incidence of DR also increases. Approximately one-third of individuals with diabetes will develop some degree of retinopathy, which currently is the leading cause of blindness in patients aged 20-74 years, in the developed and developing countries (Cheloni et al., 2019; Ruta et al., 2013).

The Italian epidemiological data available, although not representing the reality of the country, show that at least $30 \%$ of diabetics have retinopathy and that $1 \%$ is affected each year by the most severe forms of the same (Italian standards for the treatment of diabetes mellitus, 2018).

\section{Mechanisms Underlying Diabetic Retinopathy}

The microvascular circulation of the retina is predominantly affected by DM, resulting in a range of structural changes. These changes ultimately cause increased permeability and altered proliferation of endothelial cells (ECs), edema, and abnormal neovascularization (NV) of the retina, leading to vision loss (Qiaoyun Gong and Guanfang Su, 2017). The pathogenesis of DR is extremely complicated, implicating numerous different mechanisms. Like other parts of the nervous system, the retina requires a continuous supply of oxygen. 
If the circulation is completely cut off by a sudden increase of intraocular pressure (IOP) to levels above systemic arterial pressure, vision in humans is altered (Linsenmeier and Zhang, 2017).

DR causes a state of suffering in the retinal bloodstream that degenerates into a condition of retinal hypoxia (slowing down and reduced supply of oxygen to the retina). As in other body areas, when a tissue no longer receives blood and therefore oxygen, it degenerates. At the retinal level, the blood vessels become weaker, change their morphology, give rise to repeated edema and bleeding that in the long term alter the retinal tissue. This stage of retinopathy is defined as not proliferating. If these ischemic alterations are accentuated or long lasting, a process can be established whereby abnormal blood vessels form in the retina to suppress the lack of oxygen; however, the structure of these new abnormal vessels is very weak and therefore they undergo repeated bleeding processes (Cheung et al., 2010).

These abnormal blood vessels or new blood vessels proliferate and destroy the retinal tissue giving rise to fibrotic (cicatricial) processes with possible retinal breakage and detachment (proliferating retinopathy) (Sorrentino et al., 2018).

DR is based on vascular damage, caused mainly by biochemical alterations related to hyperglycemia with a consequent increase in vascular permeability, and by a high state of tissue hypoxia with overexpression of VEGF, inflammatory cytokines and oxygen reactive species (Cohen and Gardner, 2016).

Reactive oxygen species are reactive intermediates of molecular oxygen that acta is important second messengers within the cells; however, an imbalance between generation of reactive ROS and antioxidant defense systems (Fig. 1) represents the primary cause of endothelial dysfunction, leading to vascular damage in both metabolic and atherosclerotic diseases (Anna Votano et al., 2021; Incalza et al., 2018). Furthermore, new mediators of vascular damage, such as microRNAs, will be discussed.

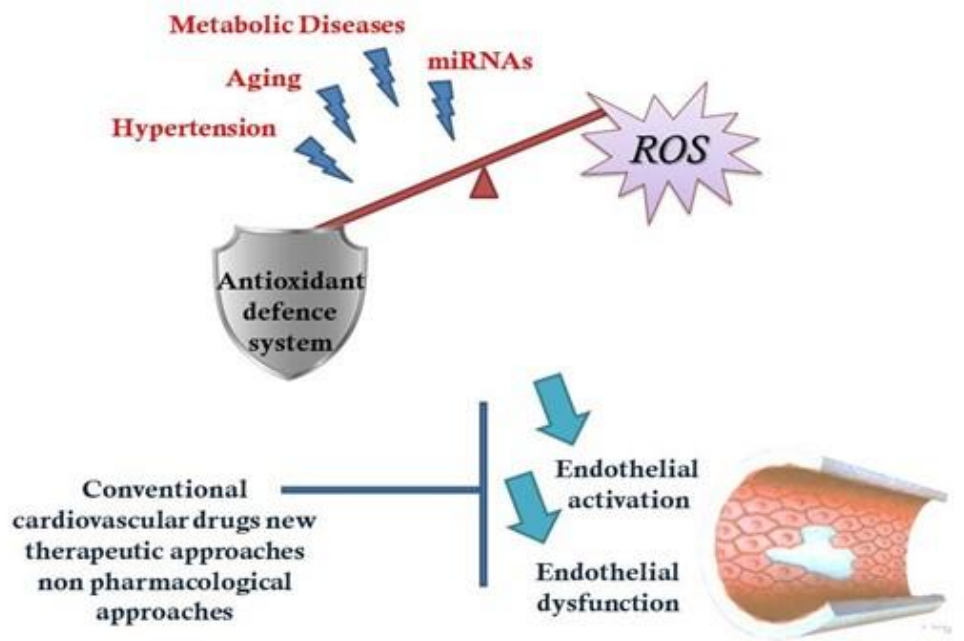

Figure 1: Reactive ROS and antioxidant defense systems: primary cause of endothelial dysfunction 
The development and progression of complications depend on the interaction between the genes, epigenetic changes due to the environment, insulin resistance, immune dysregulation and inflammation (Fig. 2). Diabetes and its complications result from common pathophysiologies.

Alteration of inflammatory responses usually occurs in the early stage of DR, which upregulates the expression of inflammatory markers, such as intercellular adhesion molecule 1 (ICAM1) and tumor necrosis factor- $\alpha$ (TNF- $\alpha$ ). Microangiopathy is due to an alteration of the capillaries, with capillary permeability, hypertrophy of the capillary cells, increase in endothelial microvilli. DR consists of a microangiopathy where small vessels are particularly sensitive to damage caused by high glucose levels. In the pathogenesis of DR hyperglycaemia is the causative factor, causing a series of reactions that include the increase in the production of catabolic products of glycosylation, the activation of the metabolism of the polyol pathway and the altered transduction of cellular signals (Yao et al., 2019). The damage to the pericytes, mesenchymal cells with contractile function that partially surrounds the endothelial cells, results in an imbalance in the mechanisms of regulation of flow controls with consequent hypoxia and accumulation of fluid inside the retinal tissue (Sinclair and Schwartz, 2019) as reported in this recent study.

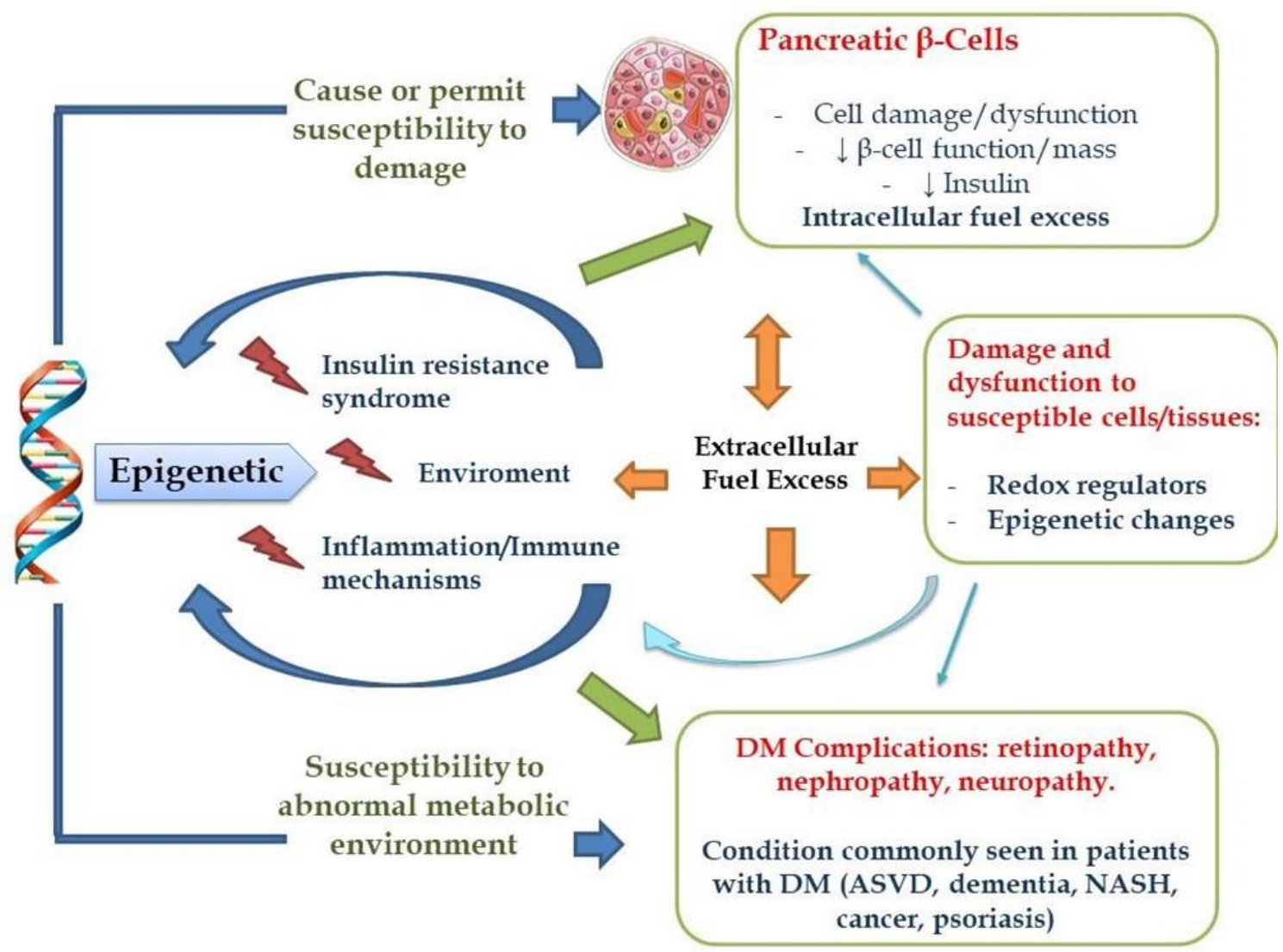

Figure 2: The developement and progression of complications depend on the interaction between the genes, epigenetic changes due to the environment, insulin resistance, immune dysregulation and inflammation 
The treatment of DR essentially involves laser therapy, which is not always decisive due to absence and/or limited availability of successful drug therapies for these blinding disorders. Currently, the main treatment for retinal neovascularization involves intravitreal injection of anti-VEGF agents, such as ranibizumab and bevacizumab (America Diabetes Association, 2013).

However, some patients show poor or no response to anti-VEGF agents with limited or no visual improvement (Kruger et al., 2013), suggesting that other molecules "e.g., PDGF and basic FGF" may be involved in retinal neovascularization (Abramsson et al., 2003).

In association with this treatment, the patient should observe adequate daily physical activity, to maintain good metabolic control, a personalized diet associated with any oral hypoglycaemic agents or insulin and drug therapy (Frank, 1997).

\section{Genes, Environmental Factors, Epigenetic Changes}

Oxidative stress induced by hyperglycaemia and glucolipotoxicity determines changes in chromatin structure that mediate changes in gene expression with consequent upregulation of proinflammatory and profibrotic mediators (Goyal et al., 2019).

These epigenetic changes are recognized as a key factor in the development and progression of diabetic vascular complications. Epigenetic modifications are generally produced by external changes (toxins, nutrients, etc.) that transduce inside cells to cause DNA methylation and histone modification which leads to an alteration of gene expression (Rodriguez and El-Osta, 2018).

In contrast with genetic modifications, epigenetic changes are susceptible to modifications by the environment as well as dysregulation over time; DNA methylation, histone modification, and non coding RNA are three major types of epigenetic effects (Feng et al., 2016; Ceriello et al., 2014). We are not aware of studies specifically examining epigenetic modulation of endothelial function in diabetes. Obviously larger scale and prospective investigations are needed to establish whether epigenetic changes affect the risk of developing diabetes in general, and endothelial dysfunction in particular (Muka et al., 2016). Altered gene expression following epigenetic changes leads to cell hypertrophy, proliferation, remodeling and apoptotic signaling and are key factors in the development and progression of diabetic complications. In fact, the expression of many genes implicated in the metabolic pathways associated with the development of diabetic retinopathy has been observed and have been increased or downgraded and changes have also been observed in the expression of microRNAs that play key roles in diabetic retinopathy, including rupture of the blood retina and the expression of neovascularization (Giovanni Faglia et al., 2013). 
It is estimated that more than $60 \%$ of protein coding genes in humans are regulated by miRNAs (Friedman et al., 2009). MicroRNAs, a class of non-coding RNAs, are important regulators of gene expression that exert their function through post-transcriptional silencing of complementary mRNA targets.

\section{MicroRNA and DR}

MicroRNAs modulate biologic processes at the post-transcriptional level by binding to the $3^{\prime}$ untranslated region ( $3^{\prime}$-UTR) of their target genes, leading to translational repression or degradation (Tang et al., 2020). Diabetic patients exhibit altered expression profiles of miRNAs involved in angiogenesis, vascular repair, and endothelial homeostasis. Of these, miRNA126 is strongly linked with vascular repair. Thus, patients with type 2 diabetes (T2DM) exhibit low levels of circulating miRNA126 compared with healthy subjects (Yi Shi and Paul, 2017).

According to recent transcriptome analyses, certain miRNA species are expressed in all retinal cell types, while others are cell type-specific. As miRNAs play important roles in homeostasis, cellular function, and survival of differentiated retinal cell types, their dysregulation is associated with retinal degenerative diseases (Zuzic et al., 2019).

In recent years, miRNAs have been shown to be involved in various biologic processes, including proliferation, differentiation, development, and metabolism, as well as in various diseases.

Some 250 miRNAs are expressed in the retina where they are components of a complex gene regulatory network (Karali et al., 2010; Krol et al., 2010; Sundermeier and Palczewski, 2012). Key processes regulated by miRNAs in the retina include adaptation to different light intensities, rapid turnover of the phototransduction cascade and maintenance of cellular homeostasis under high activity. Furthermore, miRNAs are implicated in control of cell differentiation, maturation and survival in retina (Chung et al., 2015).

Abnormal development of blood vessels within the retina plays important roles in many ocular neovascular diseases, including retinopathy of prematurity, proliferative diabetic retinopathy, and wet age-related macular degeneration. Endothelial cell proliferation and migration lead to the angiogenic growth of new blood vessels sprouting from retinal veins, and may result in vitreous hemorrhage, retinal detachment, and even blindness (Bharadwaj et al., 2013). Previous studies have shown that retinal angiogenesis is regulated by many angiogenesis-related factors, including vascular endothelial growth factor platelet-derived growth factor (PDGF), fibroblast growth factor [FGF], and transforming growth factor (Yang et al., 2016). 


\section{MicroRNA and Inhibition of Endothelial Cell Proliferation in DR}

Just like elsewhere in the body, the function of miRNAs has shown increasing relevance in the eye. In the back of the eye, the retina consists of a thin layer of neuronal tissue of diverse cell types and is equipped with a highly specialized light-sensing capacity (Liu et al., 2020) (Fig. 3). Fine-tuning gene expression for cell differentiation and function is crucial for vertebrate retinal development and proper vision.
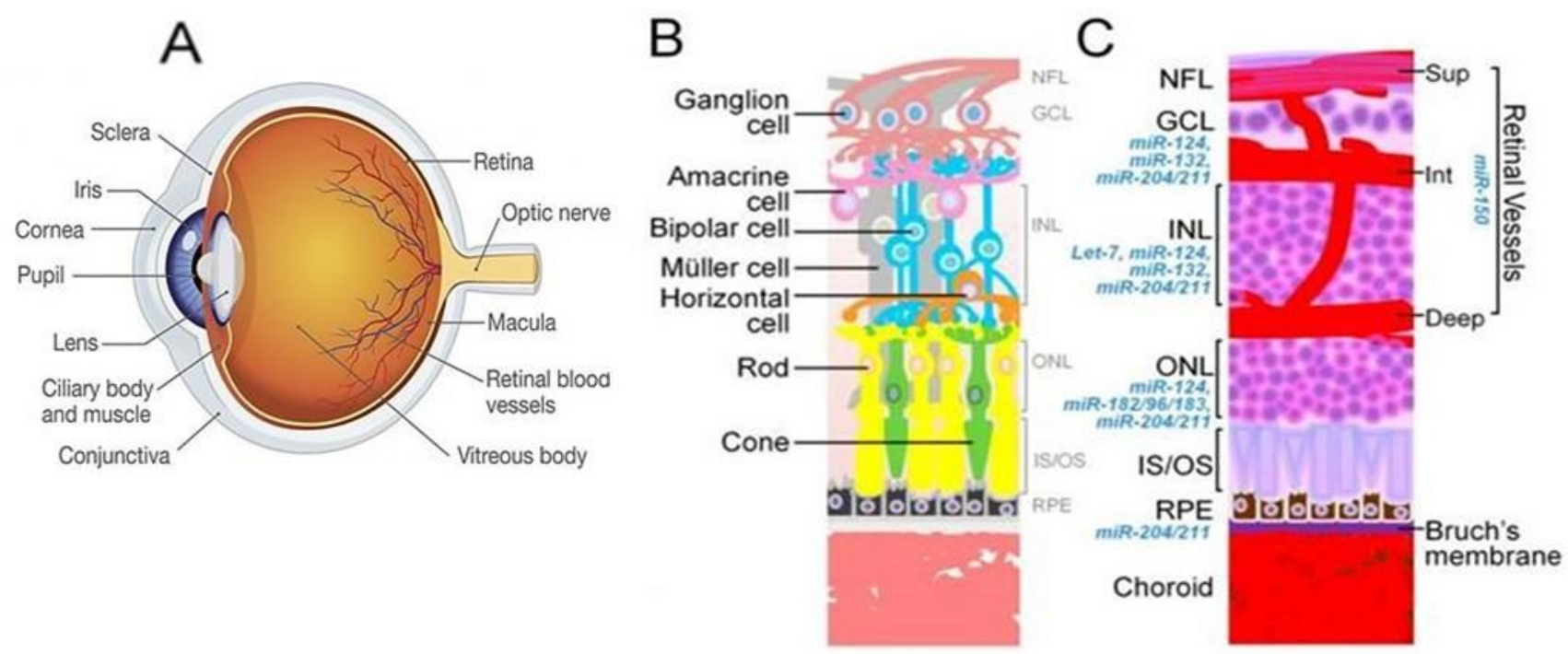

Figure 3: The Anatomy of The Eye and Relevant miRNAs

(A) The schematic diagram illustrates the main structures of the human eye. (B) The schematic representation of the cell types in the neural retina depicts their cellular connections (including ganglion cells, amacrine cells, bipolar cells, horizontal cells, as well as rod and cone photoreceptors) and supporting cells (Müller cells and RPE). (C) A cross section of the eye shows the laminar organization of the nuclear layers (GCL, INL, and ONL), the retinal vasculature, and segments of photoreceptors (IS/OS). The RPE monolayer, with Bruch's membrane underneath, is located between the neural retina and the choroid complex. miRNAs that regulate the physiological functions or pathological conditions related to each retinal neuronal and vessel layers, and RPE, are listed next to respective histological structure.

Abnormal angiogenesis disrupts the delivery of oxygen and nutrients, which can lead to an unbalanced metabolic status, and result in structural instability and functional loss of affected tissues. Excessive angiogenesis, also known as neovascularization, has considerable pathophysiologic roles in DR (Yang et al., 2020).

In some studies, several miRNAs had reported important roles in DR (Liu et al., 2018; Zhang et al., 2017). 
For example, miR-15a has been shown to regulate angiogenesis in DR through targeting VEGF. MiR451 inhibited the proliferation and migration of retinal pigment epithelial cells in proliferative DR (Li X and e Shao, 2015). MiR-200b and miR-29a have also been involved in the development of DR. MicroRNA200b is downregulated in retinal endothelial cells and cardiac microvascular endothelial cells exposed to high glucose, and its overexpression reduces VEGF expression and prevents diabetic retinopathy and cardiomyopathy (McArthur et al., 2011).

Accumulated evidence has revealed that miRNAs play important roles in endothelial cell dysfunction and angiogenesis. In this study it was seen that the miR-29B-3B inhibits the proliferation and angiogenesis of retinal microvascular endothelial cells (RMEC), while inhibition of miR-29b-3p had opposite effects. These effects of miR-29b-3p on RMEC were mediated by the negative regulation of the expression of VEGFA and PDGFB (Tang et al., 2020).

Recent study has revealed that miR-29b regulates VEGF-A expression by binding directly to their 3'-UTR (Li P et al., 2013). With this study, miR-29b-3p was found to regulate VEGF-A expression negatively, while inhibition of miR-29b-3p increased VEGF-A expression.

In this study (Chen et al., 2019) has been studied that miR-144-3p significantly inhibited cell proliferation induced by high glucose (HG) through suppressing fibroblast growth factor (FGF)16, suggesting miR-144-3p as a potential target for DR therapy. In previous studies, it has been seen that miR-144-3p was down-reguleted in rat DR model, which was further confirmed in the present study

Analysis of circulating miRNAs from serum or plasma samples of DM patients both with and without DR showed altered expression levels of many miRNAs throughout different patient populations (e.g., ages, type of DM, years after onset, etc.) (Wu JH et al., 2012).

The miRNA146 family participates in the regulation of oxidative stress and the production of proinflammatory factors. Circulating levels of miR146a are decreased in diabetic patients. Among them, miR-27b and miR-320a are the two miRNAs mostly associated with the risks of DR in T1DM (Qing et al., 2014). MiR-221, an anti-angiogenic miRNA, is significantly altered in DM and is involved in the DM physiopathology and macrovascular complications associated with T2DM (Zampetaki et al., 2016; Liu HN et al., 2018; Fiorentino et al., 2013; Costantino et al., 2016). In addition, other circulating miRNAs including miR-126 (Lightell Jr et al., 2018), miR-150 (Qin LL et al., 2017), miR-155 (Mazzeo et al., 2018), and miR-200b (Yang TT et al., 2015) are also dysregulated in DR patients, as well as in preclinical animal models of DR (Li EH et al., 2017; Ye P et al., 2014; Bai Y et al., 2011; Wang and Yan, 2016; McAuley et al., 2015). 
These findings indicate the complex regulation of miRNAs in DR and the potential of miRNAs as biomarkers and/or therapeutic targets for treating DR (Qing et al., 2014).

These results indicated significant roles of miRNA in DR and consequently we wanted to indicate the main microRNAs involved in DR.

\section{Conclusions}

A systematic search of English-language articles in PubMed database was performed using the search terms of the headings of medical subjects [MeSH] "diabetic retinopathy", "vascular endothelial growth factor", "VEGF", "ADMA", "microRNA and diabetic retinopathy", "diabetic retinopathy and endothelial dysfunction".

The research date range was limited to a period between February 2012 and January 2020, except for 7 articles. Only articles and/or experimental studies that analyzed and studied microRNAs were included; such as: miR-15a, miR-451, miR-200b, miR-29a, miR-29b-3p, miR-144-3p, miR-27b, miR-320a, miR-221, miR-150, miR-155.

During our research, only diabetic retinopathy was included as a microvascular complication of DM. Clinical cases and "gray literature" articles have been excluded.

Since the discovery of the first microRNA (miRNA) decades ago, miRNA biology studies have expanded into many fields of biomedical research, including ocular research. Although microRNAs (miRNAs) provide a recently recognized level of regulation of gene expression, the retinal miRNA transcriptome and the contribution of miRNAs to retinal development and function are largely unknown.

Therefore, in addition to diagnosing DR through histological examination of the fundus vasculature, circulating miRNAs were found to play critical roles in DR development, suggesting that miRNAs could also serve as new biomarkers in detecting or predicting the progress of retinopathy and furthermore, the overall progress of DM. Analysis of circulating miRNAs from serum or plasma samples of DM patients both with and without DR showed altered expression levels of many miRNAs throughout different patient populations, such as: ages, type of DM, years after onset, etc.

MiRNA-targeted treatments, particularly those that interfere with the balance between VEGF and NO and endothelial progenitor cell function, may be promising avenues for therapeutically addressing endothelial dysfunction in diabetes. This hypothesis requires further experiments to validate and more clinical studies to be verified. In conclusion, this study adds to the growing number of tests involving miRNA in regulating angiogenesis. To begin to understand the functions of miRNAs in the retina, we 
wanted to indicate just some of the many microRNAs involved in DR. There are several ongoing clinical studies designed to test the efficacy of miRNAs in the treatment of various disorders, so that gene therapy miRNA-based may become a new therapeutic option for a wide range of diseases in the future.

\section{Acknowledgement: The author has no conflicts of interest to report.}

\section{References}

Abramsson A, Lindblom P, Betsholtz C. Endothelial and non endothelial sources of PDGF-B regulate pericyte recruitment and influence vascular pattern formation in tumors. J Clin Invest 2003; 112: 1142.

America Diabetes Association. Microvascular Complications and Food Care: Standards of Medical Care in Diabetes - 2019. Diabetes Care 2013; 42: S124-S138.

Anna Votano, Alessia Bonofiglio, Federica Catalano, Claudia Paone. Importance of Alpha Lipoic Acid (ALA): Antidiabetic and Antioxidant Effects. Front Med Case Rep 2021; 2: 1-09.

Bai Y, Bai X, Wang Z, Zhang X, Ruan C, Miao J. MicroRNA-126 inhibits ischemia-induced retinal neovascularization via regulating angiogenic growth factors. Exp Mol Pathol 2011; 91: 471-477.

Bharadwaj AS, Appukuttan B, Wilmarth PA, Pan Y, Stempel AJ, Chipps TJ, Benedetti EE, Zamora DO, Choi D, David LL, Smith JR. Role of the retinal vascular endothelial cell in ocular disease. Prog Retin Eye Res 2013; 32: 102-180.

Ceriello A, Novials A, Ortega E, Pujadas G, La Sala L, Testa R, Bonfigli AR, Genovese S. Hyperglycemia following recovery from hypoglycemia worsens endothelial damage and thrombosis activation in type 1 diabetes and in healthy controls. Nutr Metab Cardiovasc Dis 2014; $24:$ 116-123.

Cheloni R, Gandolfi SA, Signorelli C, Odone A. Global prevalence of diabetic retinophaty: protocol for a systematic review and meta-analysis. BMJ Open 2019; 9: e022188.

Chen C, Zhao C, Gu C, Cui X, Wu J. MiRNA-144-3p inhibits high glucose induced cell proliferation through suppressing FGF16. Biosci Rep 2019; 39: BSR20181788.

Cheung N, Mitchell P. Wong TY. Diabetic retinophaty. Lancet 2010; 376: 124-136.

Chung SH, Gillies M, Sugiyama Y, Zhu L, Lee SR, Shen W. Profiling of microRNAs involved in retinal degeneration caused by selective Müller cell ablation. PLoS One 2015; 10: e0118949.

Cohen SR and Gardner TW. Diabetic Retinopathy and Diabetic Macular Edema. Dev Ophthalmol 2016; 55: $137-146$.

Costantino S, Paneni F, Lüscher TF, Cosentino F. MicroRNA profiling unveils hyperglycaemic memory in the diabetic heart. Eur Heart J 2016; 37: 572-576.

Das A and McGuire PG. Retinal and choroidal angiogenesis: pathophysiology and strategies for inhibition. Prog Retin Eye Res 2003; 22: 721-748.

Feng B, Cao Y, Chen S, Chu X, Chu Y, Chakrabarti S. MirR-200b mediates endothelial to mesenchymal transition in diabetic cardiomyopathy. Diabetes 2016; 65: 768-779.

Fiorentino L, Cavalera M, Mavilio M, Conserva F, Menghini R, Gesualdo L, Federici M. Regulation of TIMP3 in diabetic nephropathy: A role for microRNAs. Acta Diabetol 2013; 50: 965-969. 
Frank RN. Growth factors in age-related macular degeneration: pathogenic and therapeutic implications. Ophthalmic Res 1997; 29: 341.

Friedman RC, Farh KK, Burge CB, Bartel DP. Most mammalian mRNAs are conserved targets of microRNAs. Genome Res 2009; 19: 92-105.

Giovanni Faglia, Paolo Beck-Peccoz, Anna Spada. Diseases of the endocrine system and metabolism. McGraw-Hill, 2013.

Goyal D, Limesand S, Goyal R. Epigenetic responses and the developmental origins of health and disease. J Endocrinol 2019; 242: T105-119

Incalza MA, D'Oria R, Natalicchio A, Perrini S, Laviola L, Giorgino F. Oxidative stress and reactive oxygen species in endothelial dysfunction associated with cardiovascular and metabolic diseases. Vascular Pharmacology 2018; 100: 1-19.

Italian standards for the treatment of diabetes mellitus 2018.

Jack Kanski and Brad Bowling. Clinical Ophthalmology. A Systematic Approach Kanski's 8th Edition. 22 May 2015;520529.

Karali M, Peluso I, Gennarino VA, Bilio M, Verde R, Lago G, Dollé P, Banfi S. miRNeye: a microRNA expression atlas of the mouse eye. BMC Genomics 2010; 11: 1-4.

Krol J, Busskamp V, Markiewicz I, Stadler MB, Ribi S, Richter J, Duebel J, Bicker S, Fehling HJ, Schübeler D, Oertner TG. Characterizing light-regulated retinal microRNAs reveals rapid turnover as a common property of neuronal microRNAs. Cell 2010; 141: 618-631.

Kruger Falk M, Kemp H, Sorensen TL. Four-year treatment results of neovascular age-related macular degeneration with ranibizumab and causes for discontinuation of treatment. Am J Ophthalmol 2013; 155: 89-95.

Lee R, Wong TY, Sabanayagam C. Epidemiology of Diabetic Retinopathy, Diabetic Macular Edema and Related Vision Loss. Eye Vis (Lond) 2015; 2:17.

Li EH, Huang QZ, Li GC, Xiang ZY, Zhang X. Effects of miRNA-200b on the development of diabetic retinopathy by targeting VEGFA gene. Biosci Rep 2017; 37: BSR20160572

Li P, Guo W, Du L, Zhao J, Wang Y, Liu L, Hu Y, Hou Y. microRNA-29b contributes to pre-eclampsia through its effects on apoptosis, invasion and angiogenesis of trophoblast cells. Clin Sci (Lond) 2013; 124: 27-40.

Li X and e Shao Y. MiRNA-451 / ATF-2 regola la proliferazione e la migrazione delle cellule epiteliali pigmentate della retina nella retinopatia diabetica proliferativa. Oftalmolo investigativo. Visual Sci 2015; 56: 44.

Lightell Jr DJ, Moss SC, Woods TC. Upregulation of miR-221 and -222 in response to increased extracellular signalregulated kinases 1/2 activity exacerbates neointimal hyperplasia in diabetes mellitus. Atherosclerosis 2018; 269: 71-78.

Linsenmeier RA and Zhang HF. Zhang. Retinal Oxygen: from animals to humans. Prog Retin Eye Res 2017; 58: $115-151$.

Liu CH, Huang S, Britton WR, Chen J. MicroRNAs in vascular eye diseases. Int J Mol Sci 2020; $21: 649$.

Liu HN, Li X, Wu N, Tong MM, Chen S, Zhu SS, Qian W, Chen XL. Serum microRNA-221 as a biomarker for diabetic retinopathy in patients associated with type 2 diabetes. Int J Ophthalmol 2018; 11: 1889-1894.

Liu TT, Hao Q, Zhang Y, Li ZH, Cui ZH, e Yang W. Effetti del microRNA-133b sulla proliferazione e sull'apoptosi delle cellule endoteliali vascolari retiniche attraverso l'angiotensina II mediata da angiotensinogeno - chinasi extracellulare-regolata dal segnale 1/2 via di segnalazione nei ratti con retinopatia diabetica. Acta Ophthalmol 2018; 10.1111 / aos.13715. 
Masuda T, Shimazawa M, Hara H. Retinal diseases associated with oxidative stress and the effects of a free radical scavenger. Oxid Med Cell Longev 2017; 2017: 9208489.

Mayur Choudhary and Goldis Malek. Rethinking nuclear receptors as potential therapeutic targets for retinal disease. $J$ Biomol Screen 2016; 21: 1007-1018.

Mazzeo A, Beltramo E, Lopatina T, Gai C, Trento M, Porta M. Molecular and functional characterization of circulating extracellular vesicles from diabetic patients with and without retinopathy and healthy subjects. Exp Eye Res 2018; 176: 69-77.

McArthur K, Feng B, Wu Y, Chen S, Chakrabarti S. MicroRNA-200b regola le alterazioni mediate dal fattore di crescita endoteliale vascolare nella retinopatia diabetica. Diabetes 2011; 60: 1314-1323.

McAuley AK, Dirani M, Wang JJ, Connell PP, Lamoureux EL, Hewitt AW. A genetic variant regulating miR-126 is associated with sight threatening diabetic retinopathy. Diab Vasc Dis Res 2015; 12: 133-138.

Muka T, Koromani F, Portilla E, O'Connor A, Bramer WM, Troup J, Chowdhury R, Dehghan A, Franco OH. The role of epigenetic modifications in cardiovascular disease: A systematic review. Int J Cardiol 2016; 212: 174-183.

Qiaoyun Gong and Guanfang Su. Roles of miRNAs and long non coding RNAs in the progression of diabetic retinopathy. Bioscience Reports 2017; 37: BSR201711157.

Qin LL, An MX, Liu YL, Xu HC, Lu ZQ. MicroRNA-126: un promettente nuovo biomarcatore nel sangue periferico per la retinopatia diabetica. Int J Ophthalmol 2017; 10: 530-534.

Qing S, Yuan S, Yun C, Hui H, Mao P, Wen F, Ding Y, Liu Q. Serum miRNA biomarkers serve as a fingerprint for proliferative diabetic retinopathy. Cell Physiol Biochem 2014; 34: 1733-1740.

Qing S, Yuan S, Yun C, Hui H, Mao P, Wen F, Ding Y, Liu Q. Serum miRNA biomarkers serve as a fingerprint for proliferative diabetic retinopathy. Cell Physiol. Biochem 2014; 34: 1733-1740.

Rodriguez $\mathrm{H}$ and El-Osta A. Epigenetic contribution to the development and progression of vascular diabetic complications. Antioxid Redox Signal 2018; 29: 1074-1091.

Ruta LM, Magliano DJ, Lemesurier R, Taylor HR, Zimmet PZ, Shaw JE. Prevalence of Diabetic Retinopathy in Type 2 Diabetes in Developing and Developed Countries. Diabet Med 2013; 30: 387-398.

Sinclair SH and Schwartz SS. Diabetic Retinopathy-An underdiagnosed and undertreated inflammatory, neuro-vascular complication of diabetes. Front Endocrinol (Lausanne) 2019; 10: 843.

Sorrentino FS, Matteini S, Bonifazzi C, Sebastiani A, Parmeggiani F. Diabetic retinopathy and endothelin system: microangiopathy versus endothelial dysfunction. Eye (Lond) 2018; 32: 1157-1163.

Sundermeier TR and Palczewski K. The physiological impact of microRNA gene regulation in the retina. Cell Mol Life Sci 2012; 69: 2739-2750.

Tang W, Guo J, Gu R, Lei B, Ding X, Ma J, Xu G. MicroRNA-29-3p inhibits cell proliferation and angiogenesis by targeting VEGF-A and PDGFB in retinal microvascular endothelial cells. Mol Vis 2020; 26: 64-75.

Tang W, Guo J, Gu R, Lei B, Ding X, Ma J, Xu G. MicroRNS-29b-3p inhibits cell proliferation and angiogenesis by targeting VEGF and PDGFB in retinal microvascular endothelial cells. Mol Vis 2020; 26: 64-75.

Wang Y and Yan H. MicroRNA-126 contributes to Niaspan treatment induced vascular restoration after diabetic retinopathy. Sci Rep 2016; 6: 26909.

Wu JH, Gao Y, Ren AJ, Zhao SH, Zhong M, Peng YJ, Shen W, Jing M, Liu L. Altered microRNA expression profiles in retinas 
with diabetic retinopathy. Ophthalmic Res 2012; 47: 195-201.

Yang S, Zhao J, Sun X. Resistance to anti-VEGF therapy in neovascular age-related macular degeneration: a comprehensive review. Drug Des Devel Ther 2016; 10: 1857-1867.

Yang TT, Song SJ, Xue HB, Shi DF, Liu CM, Liu H. Regulatory T cells in the pathogenesis of type 2 diabetes mellitus retinopathy by miR-155. Eur Rev Med Pharmacol Sci 2015; 19: 2010-2015.

Yang Y, Liu Y, Li Y, Chen Z, Xiong Y, Zhou T, Tao W, Xu F, Yang H, Ylä-Herttuala S, Chaurasia SS. MicroRNA-15b Targets VEGF and Inhibits Angiogenesis in Proliferative Diabetic Retinopathy. The Journal of Clinical Endocrinology \& Metabolism 2020; 105: 3404-3415.

Yao Y, Du J, Li R, Zhao L, Luo N, Zhai JY, Long L. Association between ICAM-1 level and diabetic retinopathy: a review and meta-analysis. Postgrad Med J 2019; 95: 162-168.

Yau JW, Rogers SL, Kawasaki R, Lamoureux EL, Kowalski JW, Bek T, Chen SJ, Dekker JM, Fletcher A, Grauslund J, Haffner S. Global Prevalence and Major Risk Factors of Diabetic Retinopathy. Diabetes Care 2012; 35: 556-564.

Ye P, Liu J, He F, Xu W, Yao K. Hypoxia-induced deregulation of miR-126 and its regulative effect on VEGF and MMP-9 expression. Int J Med Sci 2014; 11: 17-23.

Yi Shi and Paul M. Vanhoutte. Macro and microvascular endothelial dysfunction in diabetes. Journal of Diabetes 2017; 9: 434-449.

Yun-Zheng Le. VEGF production and signalling in Müller glial are critical to modulating vascular function and neuronal integrity in diabetic retinopathy and hypoxic renal vascular diseases. Vision Res 2017; 139: 108-114.

Zampetaki A, Willeit P, Burr S, Yin X, Langley SR, Kiechl S, Klein R, Rossing P, Chaturvedi N, Mayr M. Angiogenic microRNAs Linked to Incidence and Progression of Diabetic Retinopathy in Type 1 Diabetes. Diabetes 2016; 65: $216-227$.

Zhang LQ, Cui H, Wang L, Fang X, Su S (2017) Role of microRNA-29 in the development of diabetic retinopathy by targeting the AGT gene in a rat model. Exp Mol Pathol 2017; 102: 296-302.

Zuzic M, Rojo Arias JE, Wohl SG, Busskamp V. Retinal miRNA functions in health and disease. Genes /Basel 2019; 10 : 377. 